Recepción: 04 / 09 / 2018

Aceptación: 23 / 11/2018

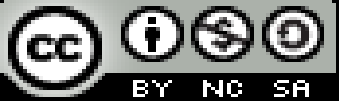

Ciencias de la salud

Publicación: 03 / 12 / 2018

Artículo científico

\title{
Características e incidencias clínicas de los pacientes pluripatológicos de la unidad de medicina interna
}

\section{Characteristics and clinical incidences of the pluripathologic patients of the internal medicine unit}

Características e incidência clínica dos pacientes pluripatológicos da unidade de medicina interna

\author{
Patricio R. Campozano-Castillo ${ }^{\mathrm{I}}$ \\ patricia_cc@gmail.com \\ María A. Delgado-López II \\ aux.dellop@gmail.com \\ Anita del R. Vera González III \\ annyveragonzalez@gmail.com
}

Correspondencia: patricia_cc@gmail.com

\footnotetext{
I Médico Cirujano, Médico General en Funciones Hospitalarias, Área Emergencia, Hospital Rodríguez Zambrano, Manta, Ecuador.

${ }^{\text {II }}$ Doctora en Medicina y Cirugía, Médico General en Funciones Hospitalarias, Área Emergencia, Hospital Rodríguez Zambrano, Manta, Ecuador.

III Magister en Gerencia y Administración en Salud, Magister en Emergencias Médicas, Doctora en Medicina y Cirugía, Médico General en Funciones Hospitalarias, Área Emergencia, Hospital Rodríguez Zambrano, Manta, Ecuador.
} 


\title{
Resumen
}

Este trabajo, tiene como objetivos determinar las características e incidencias de los pacientes pluripatológicos que sufren de forma simultánea varias enfermedades crónicas. Como método, re realizó un estudio descriptivo a partir del conjunto mínimo básico de datos (CMBD) basados en recopilación de la información publicada y disponible en las diferentes bases de datos, y de una definición funcional de paciente pluripatológico. Se recogen como resultado que aproximadamente el $38 \%$ de los ingresos en medicina interna fueron pacientes pluripatológicos. Éstos generalmente son mayores de edad, varones, que ingresan con frecuencia de forma urgente. Se concluye que los pacientes pluripatológicos presentan características diferenciales que permiten su identificación retrospectiva mediante el análisis del CMBD.

Palabras clave: Paciente pluripatológico; estudios de prevalencia; medicina interna; enfermedades crónicas.

\begin{abstract}
The objective of this work is to determine the characteristics and incidences of multi-pathological patients who suffer from several chronic diseases simultaneously. As a method, re conducted a descriptive study based on the minimum basic data set (MBDS) based on the compilation of the information published and available in the different databases, and a functional definition of a multi-pathological patient. The result is that approximately $38 \%$ of the internal medicine revenues were multi-pathological patients. These are usually of age, males, who frequently enter urgently. It is concluded that the pluripathologic patients present differential characteristics that allow their retrospective identification through the analysis of the MBDS.
\end{abstract}

Keywords: Multipathologic patient; prevalence studies; internal medicine; chronic diseases.

\section{Resumo}

O objetivo deste trabalho é determinar as características e a incidência de pacientes multipatológicos que sofrem de várias doenças crônicas simultaneamente. Como método, realizou-se um estudo descritivo baseado no conjunto mínimo de dados básicos (MBDS) baseado na compilação das informações publicadas e disponíveis nas diferentes bases de dados, e na definição funcional de um paciente multipatológico. O resultado é que aproximadamente $38 \%$ das receitas de medicamentos internos eram pacientes multipatológicos. Estes são geralmente de 
idade, os machos, que freqüentemente entram com urgência. Conclui-se que os pacientes pluripatológicos apresentam características diferenciais que permitem sua identificação retrospectiva por meio da análise da SMD.

Palavras chave: Paciente multipatológico; estudos de prevalência; medicina interna; doenças crônicas.

\section{Introducción}

El concepto de paciente pluripatológico (PPP), aún no cuenta con una definición universalmente aceptada, se ha consolidado entre los profesionales de la medicina para designar a los pacientes, en su mayoría de avanzada edad, en los que concurren varias enfermedades crónicamente sintomáticas y con frecuentes reagudizaciones, que actúan negativamente sobre su situación funcional y que generan una importante demanda en los diferentes niveles asistenciales ${ }^{1,2}$. En los hospitales de agudos de los países desarrollados el ingreso hospitalario de pacientes ancianos con comorbilidad asociada se ha convertido en los últimos años en un fenómeno creciente ${ }^{1-6}$. La prevalencia de estos pacientes se ha estudiado fundamentalmente en los servicios de medicina interna, donde aproximadamente el $38 \%$ de los pacientes son portadores de enfermedades crónicas consideradas pluripatológicas, ya que afectan a más de un órgano ${ }^{8-13}$, junto a una importante limitación funcional ya una edad media de 75 años. Para atender a estos pacientes, denominados pluripatológicos, García-Morillo et al han desarrollado en su servicio de medicina interna una unidad asistencial que les ha permitido detectar en éstos una especial fragilidad clínica y un mayor deterioro funcional. En un editorial publicado en la revista Medicina Clínica, Sánchez Rodríguez ${ }^{14}$ plantea la necesidad de una atención integral coordinada a estos pacientes, para lo que se propone una readaptación de los servicios de medicina interna que permita a los internistas realizar valoraciones funcionales, coordinar distintos niveles asistenciales y trabajar con equipos multidisciplinarios. De un modo curioso y coincidente, éstas son las tres características definitorias de la especialidad de geriatría, descritas en manuales de geriatría paraestudiantes $^{15}$ y para médicos ${ }^{16}$. En el editorial de Sánchez Rodríguez ${ }^{14}$ subyace la preocupación por reivindicar, para la medicina interna, la dirección del proceso asistencial a estos pacientes, y menciona de forma tangencial que se debería contar con la colaboración de la geriatría para determinados casos muy concretos. Creemos que la reivindicación de exclusividad 
para la medicina interna de la atención hospitalaria a los ancianos frágiles es desacertada, sobre todo cuando hay una especialidad médica hospitalaria, denominada geriatría, que comparte la opinión de que la respuesta del sistema sanitario actual no se ajusta ni a las expectativas subjetivas ni a las necesidades objetivas del paciente frágil ${ }^{17}$. Aunque también nos preocupa el futuro de la medicina interna en los hospitales, nos parece más lógico que los internistas traten de recuperar su preeminencia en la atención a los pacientes adultos en todas las plantas de hospitalización médica. Así, los subespecialistas médicos podrían dedicarse mejor al desarrollo de sus técnicas y actuarían con mayor eficiencia en la atención clínica como consultores de los internistas. Ésta es una tendencia que se observa ya en los Estados Unidos, donde se ha «reinventado» al internista denominándolo «hospitalista» ${ }^{18}$, tendencia semejante a la asunción por parte de los intensivistas de todos los pacientes ubicados en las áreas de pacientes críticos ${ }^{19}$.

\section{Métodos}

Estudio descriptivo, que utiliza como fuente de datos el conjunto mínimo básico de datos (CMBD). Este estudio se basa en información recopilada en diferentes publicaciones que constan en diversas bases de datos y en una muestra de 250 pacientes que ingresaron al Área de Medicina Interna en el Hospital Rodríguez Zambrano, de la ciudad de Manta.

Los códigos de la Clasificación Internacional de Enfermedades (CIE, 9. a revisión, modificación clínica) sirvieron para identificar inicialmente a cada paciente como PPP o paciente no pluripatológico (PNP), considerando PPP al que cumple al menos 2 de las 7 categorías en las que se agrupan las enfermedades crónicas más prevalentes en su medio. Se analizaron en cada grupo las variables demográficas (edad, sexo) y las variables de gestión: tipo de ingreso (urgente, resto), área de ingreso, servicio responsable del alta, tipo de alta (domicilio, resto) y número de estancias generadas.

Las variables continuas se expresaron como media y las variables categóricas como porcentajes; en ambos casos se estimó también el correspondiente intervalo de confianza (IC) del 95\%. Se realizó un análisis bivariante de cada una de las variables con respecto a la condición de ser o no PPP, aplicando para las variables cualitativas la prueba de la $\chi^{2}$ (o el test exacto de Fisher cuando el número de observaciones fue demasiado bajo), y para las variables cuantitativas la prueba de la t de Student. 


\section{Resultados}

El 16,1\% del total de altas hospitalarias fueron PPP. De ellos, un 28,2\% cumplía más de 2 categorías de la definición funcional ${ }^{8}$.

Existen diferencias en cuanto a las características demográficas y variables de gestión para los grupos PPP y PNP. Los PPP frecuentan mayor edad (>70 años) que los PNP, y son generalmente varones. Además, los PPP habían ingresan de forma urgente en el hospital más asiduamente que otros tipos de pacientes, y también, con más frecuencia que éstos, entrando por el área de emergencias, luego atendidos en medicina interna para luego ser derivados a las respectivas especialidades. En cuanto al destino de los pacientes en el momento del alta, los PPP son remitidos a su domicilio con menos frecuencia que los PNP. A este respecto, cerca del 13\% de los PPP son dados de alta por fallecimiento. Los PPP generan casi el doble de días de estancia hospitalaria, lo que incurre en un promedio de 14 días.

El 75\% de los PPP habían son dados de alta desde el área de especialidades médicas, y el 15\% desde el área de especialidades quirúrgicas. El servicio de medicina interna es responsable del 40\% de los PPP dados de alta en el área de especialidades médicas. A su vez, el área de especialidades quirúrgicas presenta una prevalencia de PPP cercana al 10\%, también con diferencias significativas entre sus servicios. Entre cirugía cardiovascular, cirugía general y traumatología manejan el 76\% de los PPP de esta área. En el área del resto de especialidades, responsable del $8 \%$ de todas las altas hospitalarias de PPP, la variabilidad entre servicios también es muy elevada.

\section{Discusión}

En este trabajo que corresponde a pacientes pluripatológicos o PPP, coincide con lo descrito por otros autores ${ }^{10-12}$, donde la principal característica distintiva es que la mayoría de pacientes son de edad más avanzada, con predominio de varones, ingresando más frecuentemente a través del servicio de urgencias, siendo atendidos en medicina interna para luego ser ubicados predominantemente en el área de especialidades médicas, presentan una tasa de mortalidad intrahospitalaria más alta que los demás pacientes, con un menor grado de alta y generan un mayor número de estancias durante su ingreso. 
Este trabajo se ha basado en una definición funcional de PPP concebida por Ollero et $\mathrm{al}^{8}$ para su aplicación de forma prospectiva, con el propósito de reconocer a una población de pacientes, con una importante fragilidad clínica y discapacidad funcional, sobre la que aplicar un proceso asistencial previamente establecido. Sin embargo, los resultados demuestran que, aplicada de forma retrospectiva y a partir únicamente de la información aportada por el CMBD, tal definición también es una buena herramienta para diferenciar, en el conjunto de un hospital universitario, a este peculiar grupo de pacientes.

La mayoría de estudios realizados en nuestro país en el ámbito hospitalario y basados en esta misma definición funcional de PPP, se han desarrollado en los servicios de medicina interna. En tal sentido, en este estudio, el servicio de medicina interna mostró un porcentaje de PPP similar al publicado por otros autores ${ }^{8,10-12}$. Pero como también pone de manifiesto esta investigación, los PPP no sólo fueron dados de alta en el servicio de medicina interna sino también en otros servicios del hospital, fundamentalmente del área de especialidades médicas y, en menor medida, en el área de especialidades quirúrgicas.

\section{Referencias Bibliográficas}

1. Sager MA, Rudberg MA, Jalaluddin M, Franke T, Inouye SK, Landefeld CS, et al. Hospital admission risk profile (HARP): identifying older patients at risk for functional decline following acute medical illness and hospitalization. J Am Geriatr Soc. 1996;44:251-7.

2. Ollero M, Cabrera JM, de Ossomo M, De Villar E, García D, Gómez E, et al. Atención al paciente pluripatológico: proceso asistencial integrado. Sevilla: Consejería de Salud, Junta de Andalucía; 2002.

3. Cesari M, Onder G, Russo A, Zamboni V, Barillaro C, Ferrucci L, et al. Comorbidity and physical function: results from the aging and longevity study in the Sirente geographic area (ilSIRENTE Study). Gerontology. 2006;52:24-32.

4. Ko Y, Coons SJ. An examination of self-reported chronic conditions and health status in the 2001 Medicare Health Outcome Survey. Curr Med Res Opin. 2005;21:1801-8.

5. Westert GP, Satariano WA, Schellevis FG, Van den Bos GAM. Patterns of comorbidity and the use of health services in the dutch polulation. Eur J Pub Health. 2001;11:365-72. 
6. Menotti A, Mulder I, Nissinen A, Giampaoli S, Feskens EJ, Kromhout D. Prevalence of morbidity and multimorbidity in elderly male populations and their impact on 10-year all-cause mortality: the FINE study (Finland, Italy, Netherlands, Elderly). J Clin Epidemiol. 2001;54:6806.

7. Matorras P, Valero MC, Zarrabeitia R, Cano M, Iglesias L, Ledesma F. Cambios y tendencias en medicina interna 1987-1996. An Med Interna (Madrid). 2000;17:295-302.

8. Starfield B, Lemke KW, Herbert R, Pavlovich WD, Anderson G. Comorbidity and the use of primary care and specialist care in the elderly. Ann Fam Med. 2005;3:215-22.

9. Estudio Socioprofesional sobre la Medicina Interna en España: Prospectiva 2010. Disponible en: http://www.fesemi.org/publicaciones/otras/index.php

10. García-Morillo JS, Bernabeu-Wittel M, Ollero-Baturone M, Aguilar-Guisado M, RamírezDuque N, González de la Puente MA, et al. Incidencia y características clínicas de los pacientes con pluripatología ingresados en una unidad de medicina interna. Med Clin (Barc). 2005;125:5-9.

11. Zambrana JL, Velasco MJ, Díez F, Cruz G, Martín MD, Adarraga MD. Características clínicas diferenciales de los enfermos pluripatológicos hospitalizados en servicios de medicina interna. Rev Clin Esp. 2005;205:413-7.

12. Pérez-Díaz JM, Martín Pérez M, Ramos-Cantos MC, SanRomán-Terán CM. Pacientes con pluripatología ingresados en una unidad de medicina interna. Med Clin (Barc). 2005; 126:37.

13. Fernández-Miera MF, Esclada Sarabia C, Sampedro García I. Manejo del paciente pluripatológico en una unidad de hospitalización domiciliaria. Med Clin (Barc). 2005;126:37-38.

14. Sánchez Rodríguez A. El enfermo con pluripatología: la necesidad de una atención integral coordinada. Med Clin (Barc). 2005;125:12-3.

15. Macías Núñez JF, editor Geriatría desde el principio. Barcelona: Glosa; 2001.

16. Guillén Llera F, Ruipérez Cantera I. Manual de geriatría. Barcelona: Masson; 2002. 
17. Alonso T, Alonso-Ruiz MT, Arana A, Gallego R, Gálvez N, Lozano G, et al. Necesidad del desarrollo de servicios especializados de atención al anciano frágil (II). Rev Esp Geriatr Gerontol. 2004;39:122-38

18. Wachter RM. Hospitalists in the United States -mission accomplished or work in progress? N Engl J Med. 2004;350:1935-6

19. Wachter RM. The evolution of the hospitalist model in the United States. Med Clin N Am. 2002;86:687-706 